\title{
Nilotinib, a discoidin domain receptor 1 (DDR1) inhibitor, induces apoptosis and inhibits migration in breast cancer
}

\author{
Shuai WANG ${ }^{1}$, Yanping $\mathrm{XIE}^{1}$, Aina $\mathrm{BAO}^{1}$, Jing $\mathrm{LI}^{1}$, Tingting $\mathrm{YE}^{1}$, Chu YANG ${ }^{1}$, Shufang $\mathrm{YU}^{2, *}$ \\ ${ }^{1}$ Ningbo First Hospital, Ningbo, China; ${ }^{2}$ Zhejiang Pharmaceutical College, Ningbo, China \\ *Correspondence: $710309357 @ q q . c o m$
}

Received November 26, 2020 / Accepted May 5, 2021

\begin{abstract}
Overexpression of discoidin domain receptor 1 (DDR1) is known to enhance the malignancy of breast cancer considerably. This study reports the identification of a potent DDR1 inhibitor, nilotinib, for the treatment of breast cancer. MTT assay was used to evaluate the inhibitory activity of nilotinib and meantime we used flow cytometry to evaluate the pro-apoptotic activity of nilotinib in MCF-7 and MDA-MB-231 cells. Expression of DDR1 was manipulated in MDA-MB-231 and MCF-7 cell lines with low-level DDR1 expression by transfecting with plasmids containing shRNA. The effect of DDR1 or treatment with nilotinib on cell migration was assayed. The expression of p-DDR1, DDR1, p-ERK1/2, ERK1/2 and E-cadherin, Vimentin, Snail1, and caspase 3 were detected by western blot and immunofluorescent staining. Nilotinib in MCF-7 $\left(\mathrm{IC}_{50}=0.403 \mu \mathrm{M}\right)$ and MDA-MB-231 $\left(\mathrm{IC}_{50}=0.819 \mu \mathrm{M}\right)$ also indicated induced apoptotic cell death. After co-culturing with nilotinib ( $500 \mathrm{nM}$ ), apoptosis rate is $29.60 \pm 2.19 \%$ and $18.75 \pm 2.30 \%$, respectively. Moreover, nilotinib effectually blocked the cellular migration of MCF-7 cells. Interestingly, the knock-down DDR1 could significantly block the migration of breast cancer, while the sensitivity of MCF-7 and MDA-MB-231 cells to nilotinib was reduced. Targeting DDR1 therapeutically could potentially affect survival and influence metabolism in breast cancer, and nilotinib could be used as a candidate for the treatment of breast cancer.
\end{abstract}

Key words: breast cancer, discoidin domain receptor, nilotinib, apoptosis, metastasis

Breast cancer is the most common type of malignancy in women and is the second leading cause of female cancer mortality in the world [1-3]. Recent reports have revealed that breast cancer is responsible for $6 \%$ of the total 7.5 million cancer mortalities worldwide [3, 4]. Although numerous advanced diagnostic and therapeutic methods have been used in the treatment of breast cancer, its metastasis is still the primary cause of death $[5,6]$.

Several studies have demonstrated the critical role played by collagen in cellular proliferation, adhesion, migration, and invasion in breast carcinoma [7, 8]. Discoidin domain receptors (DDRs) are a family of receptor tyrosine kinases (RTKs), which are activated in response to collagen, and are composed of two highly homologous subtypes, DDR1 and DDR2 [9, 10]. DDR1 is known to play a vital role in breast cancer metastases [11]. Overexpression of DDR1 is associated with a poor prognosis of breast cancer, sometimes even lethal, in the patients $[12,13]$. Activation of DDR1 by collagen IV induces the MAPK signaling pathway, thus promoting the proliferation of malignant breast cancer cells $[14,15]$. However, DDR1 is also known to stabilize E-cadherin and facilitate cellular migration by suppressing actomyosin at the sites of cellcell contact $[16,17]$. Thus, blocking the phosphorylation of DDR1, resulting in its reduced expression might be an effective strategy for the treatment of breast cancer.

Several reports have verified that nilotinib is a potent DDR1 inhibitor. Clinical studies have also approved its use as a BCR-ABL kinase inhibitor [18-20]. It has been reported that nilotinib can treat chronic myeloid leukemia (CML) by inhibiting DDR1 phosphorylation [21]. Additionally, nilotinib has been identified as a brand-new method for the treatment of metastatic colorectal cancer by the inhibition of the DDR1-BCR signaling pathway $[22,23]$ and nilotinib also has been reported as an ABL kinase inhibitor for blocking breast cancer metastasis [23]. However, there are no reports on the evaluation of nilotinib as a novel DDR1 inhibitor for the treatment of breast cancer. In the present study, our main aim is to illuminate the biological role of the DDR1 signaling pathway in breast carcinoma and to evaluate the inhibitory activity of nilotinib against breast cancer. 


\section{Materials and methods}

Cell culture. Human breast cancer cell lines MCF-7 and MDA-MB-231 (obtained from ATCC) were grown in DMEM (GIBCO) containing 10\% FBS (GIBCO). The cell lines were maintained at $37^{\circ} \mathrm{C}$ in a $5 \% \mathrm{CO}_{2}$ incubator.

MTT assay. MCF-7, MDA-MB-231, MCF-7 SHDDR1, and MDA-MB-231 SHDDR1 cells were separately cultured in $10 \%$ FBS growth medium in 96-well plates (5,000 cells/well) overnight. The cells were treated with various concentrations of nilotinib ( $\mathrm{n}=11 ; 14.5-30,000 \mathrm{nM})$ and cultured in 10\% FBS medium for $48 \mathrm{~h}$ in triplicates. The control cells were treated with dimethyl sulfoxide (DMSO) only. Next, tetrazolium dye (MTT) solution ( $5 \mathrm{mg} / \mathrm{ml}, 20 \mu \mathrm{l} /$ well) was added to each well. After incubation for $4 \mathrm{~h}$ at $37^{\circ} \mathrm{C}$, the supernatant was aspirated, the generated formazan crystals were dissolved in $150 \mu \mathrm{l}$ of DMSO, and the absorbance was recorded spectrophotometrically at $490 \mathrm{~nm}$ using an enzyme-linked immunosorbent assay plate reader. The data were calculated using GraphPad Prism version 5.0. The fitting of the $\mathrm{IC}_{50}$ values was done using a non-linear regression model with a sigmoidal dose-response.

Cell apoptosis assay. MCF-7 and MDA-MB-231 $\left(3 \times 10^{5}\right)$ cells were seeded into 6-well plates overnight. Fresh growth media with nilotinib (500 $\mathrm{nM})$ as well as medium with $1 \%$ DMSO (control) was added to the plates. After $48 \mathrm{~h}$, the growth medium was collected, and cells were trypsinized and collected with the corresponding medium. After centrifugation at $1,000 \times \mathrm{g}$ at $4^{\circ} \mathrm{C}$ for $3 \mathrm{~min}$, the supernatant was removed completely, and the cells were washed twice with cold PBS. Then, $100 \mu \mathrm{l}$ of $1 \times$ binding buffer, $5 \mu \mathrm{l}$ PI (PI, BD) and $5 \mu$ Annexin-V (FITC-Annexin V, BD) were added. The cells were then gently vortex-mixed and incubated for $15 \mathrm{~min}$ at room temperature in the dark and $1 \times$ binding buffer was used for dilution to $500 \mu \mathrm{l}$. The cells were then stained with PI, and Annexin-V alone as a positive control. The samples were measured using a BD Accuri ${ }^{\mathrm{Tx}}$ C6 flow cytometer (Becton Dickinson), and the data were processed using FlowJo 7.6.1.

Western blotting. Protein extracts were obtained using the KEYGEN total protein extraction kit (Nanjing, China). The concentrations of protein in the supernatant fractions were determined using a BCA Protein Assay Kit (Pierce). Sixty micrograms of protein per sample were loaded and separated on sodium dodecyl sulfate-polyacrylamide gel electrophoresis (SDS-PAGE), followed by electrophoretic transfer onto a polyvinylidene fluoride (PVDF) membrane (Millipore, Bedford, MA, USA). After blocking with 5\% skim milk in Tris-buffered saline containing $0.05 \%$ Tween-20, the membrane was first incubated with the indicated primary antibody at $4{ }^{\circ} \mathrm{C}$ overnight. Signals were detected using an ECL+TM Western blotting system (Bio-Rad, Hercules, CA). Primary antibodies used were: anti-phospho-DDR1 (cst\#11994), anti-DDR1 (cst\#5583), anti-ERK1/2 (cst\#9194), anti-phospho-ERK (cst\#4370), E-cadherin (cst\#14472),
Vimentin (cst\#5741), cleaved caspase-3 (cst\#9664), and antiGAPDH (cst\#5174) from Cell Signaling Technology and Snail 1 (\#14-9859-82) from Thermo Fisher Scientific.

Wound-healing assay. MCF-7 SHctrl and MCF-7 SHDDR1, MDA-MB-231 SHctrl and MDA-MB-231 SHDDR 1 cells were seeded in 6-well plates and incubated at $37^{\circ} \mathrm{C}$ overnight. After reaching $100 \%$ confluence, a straight line of similar width was scratched across the monolayer using a $10 \mu \mathrm{l}$ pipette tip, for each sample. After washing with PBS to remove non-adherent cells, cells were then treated with the indicated concentrations of nilotinib and incubated for $24 \mathrm{~h}$. When the wound in the DMSO control was healed, the image from the original scratch line was captured by Leica DFC 3000 G.

Immunofluorescence staining. Cells were seeded in a confocal dish, fixed with ice-cold $4 \%$ paraformaldehyde, and incubated with $0.5 \%$ bovine serum albumin in phosphatebuffered saline (PBS) with $0.1 \%$ Triton X-100 for $30 \mathrm{~min}$. After washing, cells were incubated with anti-phosphoDDR1 (\#PA5-106123, Thermo Fisher Scientific) antibody before rinsing with PBS, then immunostained with secondary antibodies, Alexa Fluor 488-goat anti-rabbit IgG (ab150077, Abcam) and stained with 4,6-diamidino2-phenylindole (DAPI) (2 $\mathrm{mg} / \mathrm{ml})$. Fluorescence images were captured using a confocal microscope (Leica TCS SP2, Germany).

Lentiviral production and transduction. For viral creation, DDR1 shRNA was designed based on the DDR1 mRNA sequence (GenBank accession no. NM-001954), the SHDDR1\#1 sequence was CCTATACGTTTCTGTGGAGTA, and SHDDR1\#2 sequence was TGCTGACATGAAGGGACATTT. Each of these sequences was cloned into a plko.1 lentiviral vector. The digestion analysis of restriction endonuclease confirmed the recombinant vector, and all inserted sequences were verified by DNA sequencing. Lentiviruses were developed by triple transfection of $80 \%$ confluent 293 T cells with DDR1 shRNAexpressing vector and the virion-packaging elements (pVSVG-I and pCMVAR8.92) using Lipofectamine 2000 (Invitrogen). They were harvested in a serum-free medium after $48 \mathrm{~h}$ and filtered through a $0.45 \mu \mathrm{m}$ filter (Millipore, Bedford, MA). Retroviruses harboring shRNA sequence were transduced into MCF-7 and MDA-MB-231 cells. After incubation for $48 \mathrm{~h}$, the transduced cells were positively selected in puromycin $(1 \mu \mathrm{g} / \mathrm{ml})$, passaged, harvested, and named MCF-7 SHDDR1\#1 and MCF-7 SHDDR1\#2, MDA-MB-231 SHDDR1\#1 and MDA-MB-231 SHDDR1\#2. Control shRNA lentiviral particles were obtained from Santa Cruz Biotechnology.

Statistical analysis. Statistical analyses were conducted using GraphPad Prism 5 software. Differences were analyzed using one-way analysis of variance followed by Tukey's post hoc test for multiple comparisons and Student's t-test for two comparisons. Data were presented as the means $\pm \mathrm{SD} .{ }^{*},{ }^{* *}$, and ${ }^{\star * *}$ indicates $\mathrm{p}<0.05, \mathrm{p}<0.01$, and $\mathrm{p}<0.001$, respectively. 


\section{Results}

Nilotinib manifests anti-proliferation and pro-apoptosis activity in breast cancer. Cytotoxicity of nilotinib in breast cancer cell lines was assessed by the MTT assay. The breast cancer cells were exposed to different concentrations of nilotinib for $48 \mathrm{~h}$. As shown in Figure 1A, the cell viabilities of MCF-7 and MDA-MB-231 cells were prominently decreased by nilotinib in a dose-dependent manner, with $\mathrm{IC}_{50}$ values of $0.403 \mu \mathrm{M}$ and $0.819 \mu \mathrm{M}$, respectively. Also, we investigated whether nilotinib inhibited the colony formation capability of breast cancer cells, a part of the tumor proliferation process. It was evident that nilotinib effectively inhibited the growth capacity of MCF-7 cells. As shown in Figure 1B, the MCF-7 and MDA-MB-231 cells exhibited a high sensitivity to the nilotinib treatment even at a low concentration of $0.1 \mu \mathrm{M}$.

Then we measured the apoptotic cell death by nilotinib with Annexin V-FITC/PI staining. As shown in Figures 1C and $1 \mathrm{D}$, the rate of apoptosis in MCF-7 and MDA-MB-231 cells, when treated with nilotinib was $29.6 \%$ and $18.8 \%$, respectively. This indicated that nilotinib could promote cellular apoptosis in both MCF-7 and MDA-MB-231 cell lines. Furthermore, we found that nilotinib promoted MCF-7 and MDA-MB-231 cells apoptosis via caspase-3 (Figure 1E), nilotinib modulated the level of cleaved caspase-3.

Nilotinib inhibits breast cancer migration. To further characterize nilotinib, its effect on cellular migration was evaluated through a wound-healing assay in the breast cancer cell line (MCF-7). Significant blocking of the cellular migra-
A

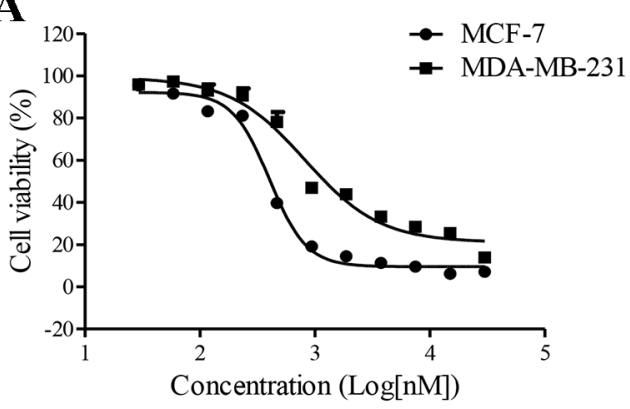

C
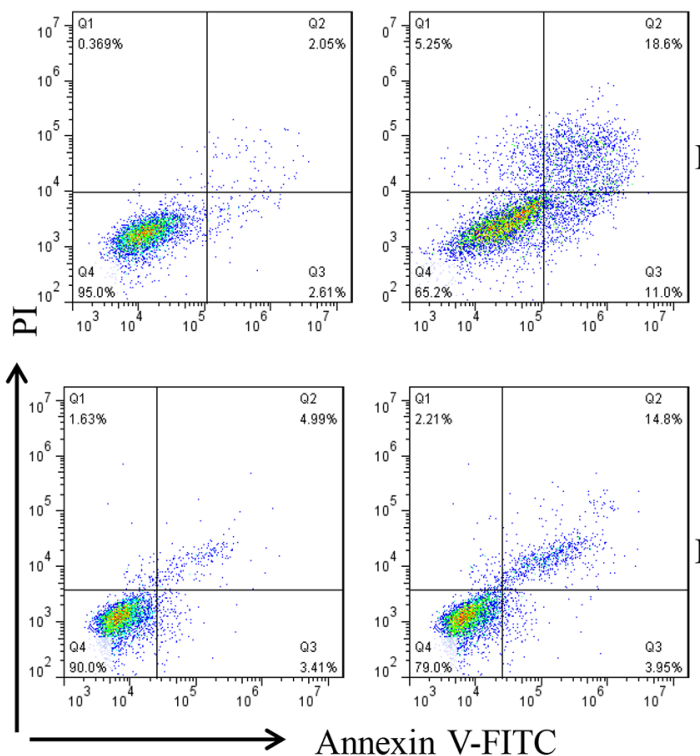

B
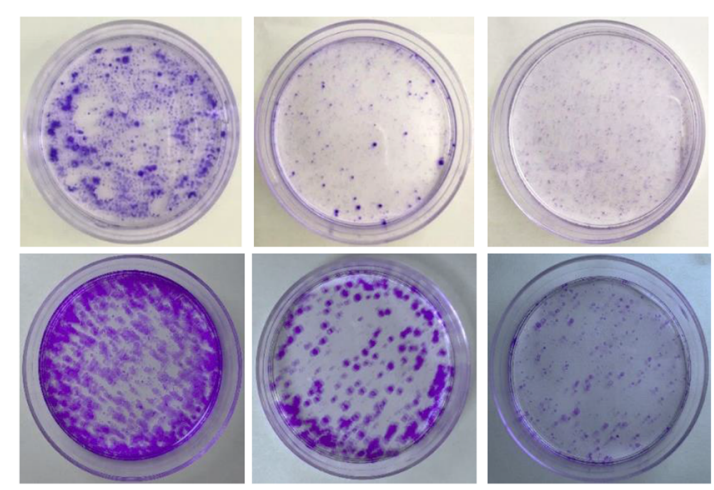

MCF-7

D

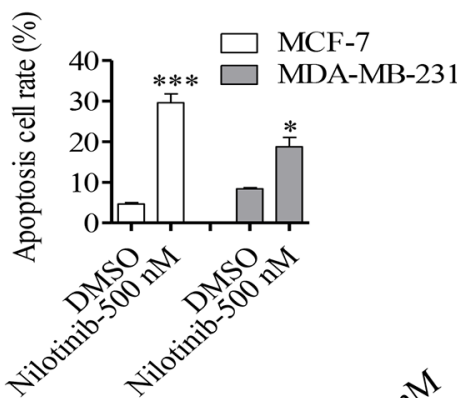

MCF-7

MDA-MB-231

MDA-MB-231

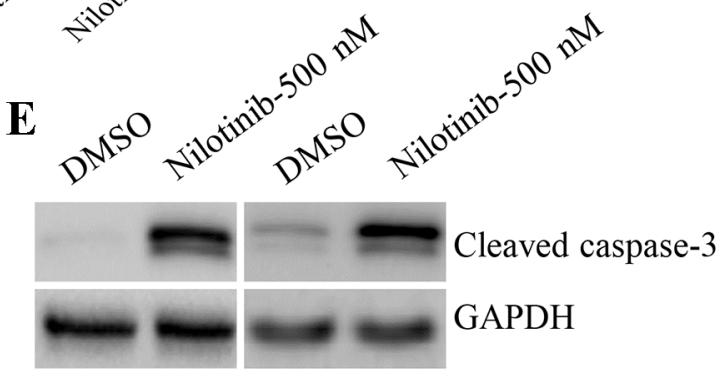

MCF-7 MDA-MB-231

Figure 1. Nilotinib manifests anti-proliferation and pro-apoptosis activity in breast cancer. A) MTT assay. The cells were treated with various concentrations of nilotinib $(\mathrm{n}=11 ; 14.5-30,000 \mathrm{nM})$ and cultured in $10 \%$ FBS medium for $48 \mathrm{~h}$ in triplicates. B) Colony formation assay. The number of colonies were counted after MCF-7 and MDA-MB-231 cells were incubated with nilotinib (100, 500 nM) for a week, and stained with crystal violet. C) Flow cytometry. D) Effects of nilotinib ( $500 \mathrm{nM})$ on apoptosis in MCF-7 and MDA-MB-231 cell lines. E) Western blot analysis for cleaved caspase-3 on MCF-7 and MDA-MB-231 cells after treatment with nilotinib $(500 \mathrm{nM}) .\left({ }^{*} \mathrm{p}<0.05,{ }^{* *} \mathrm{p}<0.01\right.$, and ${ }^{\star * *} \mathrm{p}<0.001$ compared to DMSO group). 
tion was observed at two concentrations ( $50 \mathrm{nM}$ and $100 \mathrm{nM}$ ) of nilotinib as compared to the DMSO group (Figures 2A, $2 \mathrm{~B})$. All the chosen concentrations $(<100 \mathrm{nM})$ exhibited low levels of cytotoxicity. Therefore, nilotinib inhibited wound healing mainly by the inhibition of cellular migration instead of blocking cell proliferation.

Vimentin is an intermediate cell filament protein that is commonly considered as a mesenchymal marker, Snail1 plays an important role in embryonic development, tumor invasion, and metastasis, and E-cadherin is as an epithelial marker. After the wound-healing assay, total protein of the cells was collected, and the level of expression of E-cadherin,
Vimentin, and Snail1 was evaluated. As shown in Figures 2C and 2D, after treatment with nilotinib, levels of E-cadherin increased, Vimentin was almost not expressed in cells, and the expression of Snaill was inhibited. This implied that nilotinib could reverse EMT (epithelial-mesenchymal transition) in breast cancer.

Nilotinib targeted DDR1 and inhibited the phosphorylation of DDR1, MAPK signaling pathway. All the experimental results indicated the inhibitory potential of nilotinib towards breast cancer, but the mechanism of action was still unknown. Previous reports have revealed nilotinib to be a potent inhibitor of DDR1. Therefore, the content of the
A
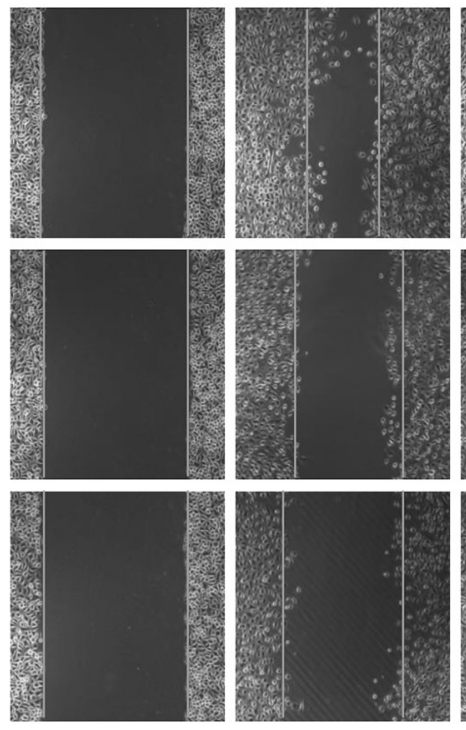

C

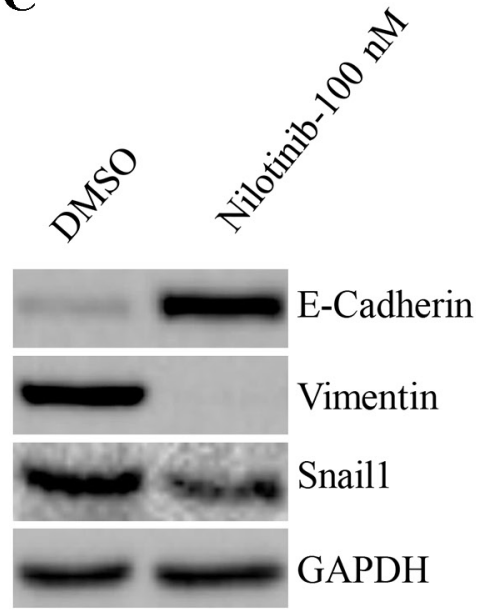

$8 \mathrm{~h}$
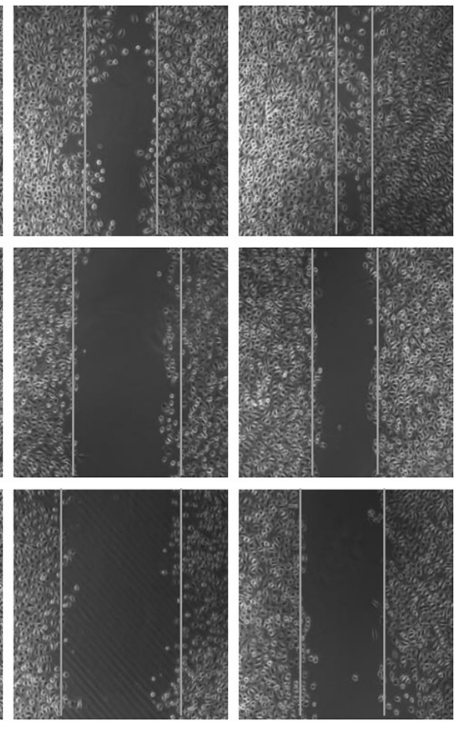

$16 \mathrm{~h}$
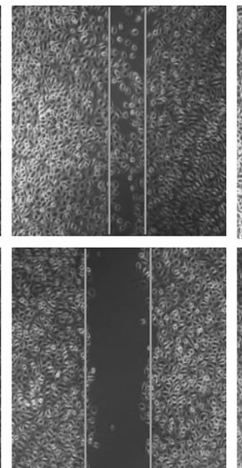

$24 \mathrm{~h}$

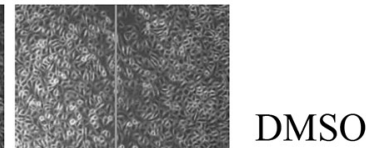

DMSO
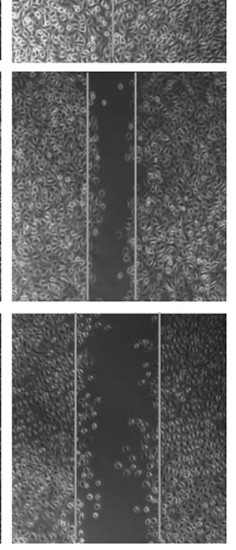

B

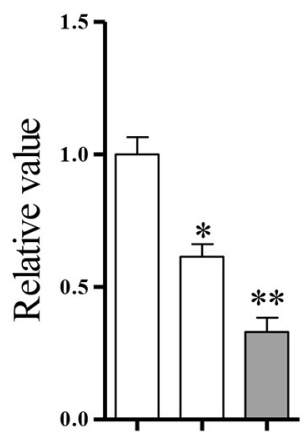

Nilotinib-100 nM

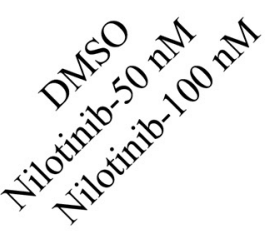

D
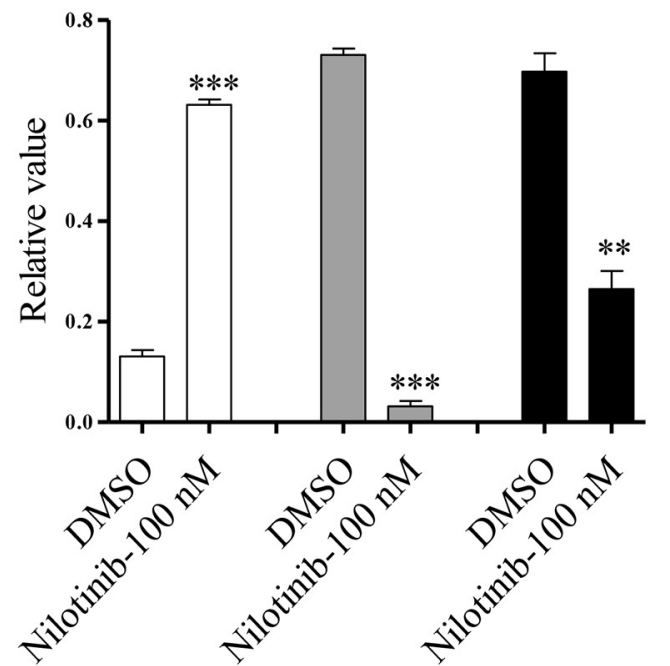

Figure 2. Nilotinib inhibits breast cancer migration. A) Wound-healing assay. MCF-7 cells were treated with two different concentrations of nilotinib $(50$ and $100 \mathrm{nM})$. B) The figures are representative of more than three separate experiments of (A). C) Western blotting. Effects of nilotinib (100 nM) on protein expression levels of E-cadherin, Vimentin, and Snail1 in MCF-7 cell line. D) The figures were representative of more than three separate experiments of $(\mathrm{C}) .\left({ }^{*} \mathrm{p}<0.05,{ }^{* *} \mathrm{p}<0.01\right.$, and ${ }^{* * *} \mathrm{p}<0.001$ compared to DMSO group). 
A

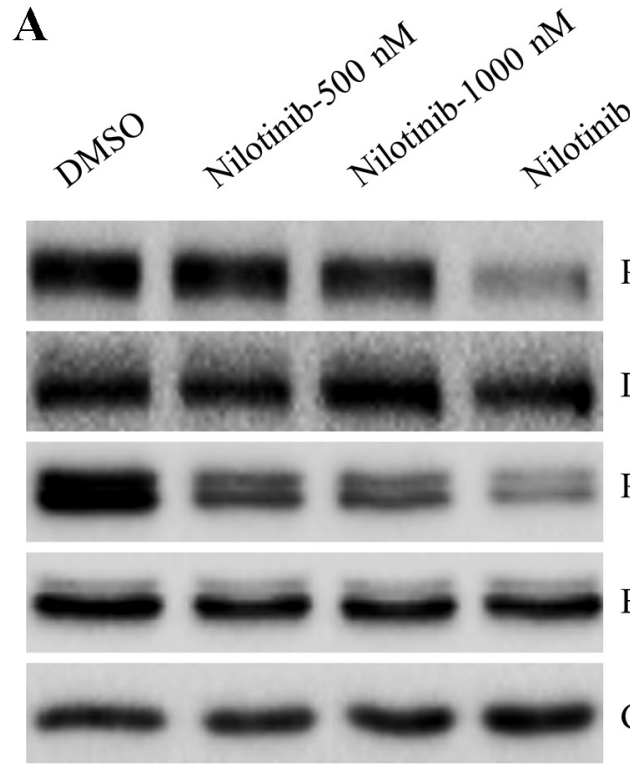

B

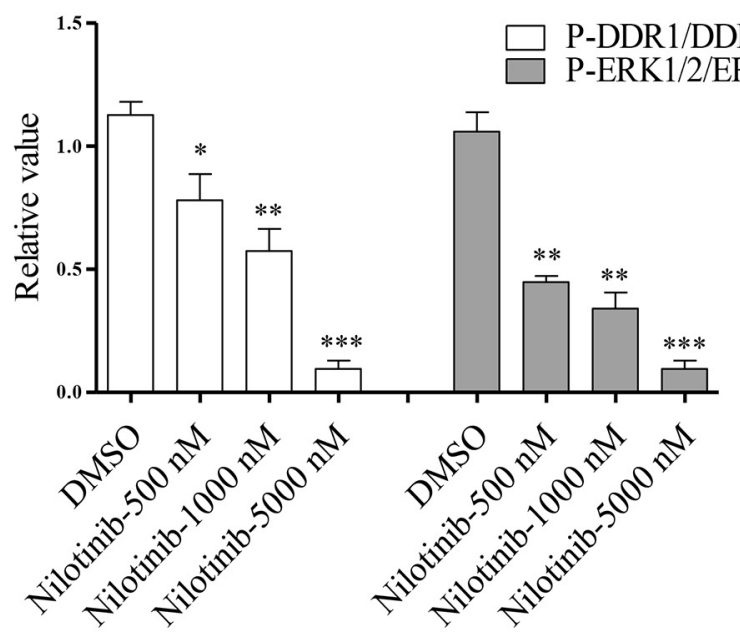

Figure 3. Nilotinib inhibits the phosphorylation of the DDR1 and MAPK signaling pathway. A) Inhibitory effect of nilotinib on DDR1 and its downstream ERK1/2 phosphorylation levels in MCF-7 cell line. B) The figures were representative of more than three separate experiments of $(\mathrm{A})$. $\left({ }^{*} \mathrm{p}<0.05\right.$, ${ }^{* *} \mathrm{p}<0.01$, and ${ }^{\star * *} \mathrm{p}<0.001$ compared to DMSO group).

phosphorylation of DDR1 protein was tested (Figures 3A, 3B). As expected, it was revealed that after treatment with nilotinib (for $3 \mathrm{~h}$ ), phosphorylation of DDR1 protein decreased in a dose-dependent manner. Further investigation along the downstream pathway revealed that the MAPK signaling pathway was blocked.

As shown in Figure 4, immunofluorescence was used to detect the level of expression of proteins in the phosphorylation of DDR1 in MCF-7 cells after treatment with nilotinib (for $3 \mathrm{~h}$ ). Three different concentrations of nilotinib (500 nM, $1000 \mathrm{nM}$, and $5000 \mathrm{nM}$ ) were chosen, to further verify the inhibitory activity of nilotinib on DDR1 phosphorylation. It was revealed that nilotinib targeted DDR1 to inhibit its activity.

DDR1 is required for migration of breast cancer and nilotinib targets DDR1 to inhibit proliferation in breast cancer. To examine whether the targeted downregulation of DDR1 in MDA-MB-231 and MCF-7 cells affected their migration and proliferation, genetic knockdown of $D D R 1$ via lentiviral shRNA-mediated RNA interference was performed. As shown in Figure 5A and Supplementary Figure S1A, levels of DDR1 protein were significantly decreased in MDA-MB231 SHDDR1 and MCF-7 SHDDR1 cells as compared to the control. In order to investigate whether nilotinib targeted DDR1 to inhibit the growth of MDA-MB-231 and MCF-7 cells, both MDA-MB-231 SHDDR1 and MCF-7 SHDDR1 cells were treated with nilotinib. As shown in Figure 5B and Supplementary Figure S1B, nilotinib had no effect on both MDA-MB-231 SHDDR1 and MCF-7 SHDDR1 cells. Also, as shown in Supplementary Figure S2, nilotinib could

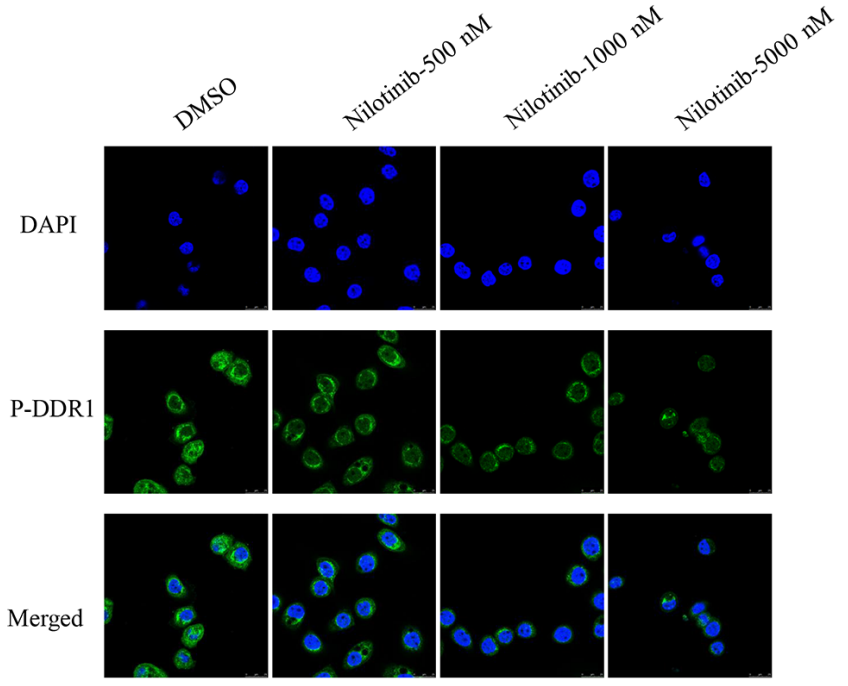

Figure 4. Immunofluorescence assay. MCF-7 cells were treated with DMSO, nilotinib $(0.5,1$, and $5 \mu \mathrm{M})$ for $3 \mathrm{~h}$, staining with the P-DDR1 antibody. Scale bars: $50 \mu \mathrm{m}$

not promote cellular apoptosis in MDA-MB-231 SHDDR1 cells. Next, migration was compared between controls to MCF-7 SHDDR1 and MDA-MB-231 SHDDR1 cells via the wound-healing assay. Interestingly, knockdown DDR1 could effectively block migration (Figure 5C, Supplementary Figure S1C). Then, total protein extraction from cells, as shown in Figure 5D and Supplementary Figure S1D, and it was observed that the expression levels of E-cadherin 
increased, while the expression levels of Vimentin and Snaill decreased. These results are consistent with the results from treatment with nilotinib.

\section{Discussion}

Metastasis is a sequential process that allows cancer cells to move from the primary tumor site and grow elsewhere in the human body $[5,24]$. This is still the leading cause of death in patients with breast cancer $[25,26]$. DDR1, a kind of receptor tyrosine kinase, is overexpressed in several malignant tumors and plays a vital role in cancer progression and metastasis [11]. Nilotinib has been reported as a DDR1 inhibitor and Jeitany et al. [20] demonstrated an additional important DDR1 kinase-dependent function in CRC metastasis formation. Moreover, they reported that pharmacological inhibition of DDR1 by nilotinib also inhibits the metastatic behavior of the CRC cells. Thus, the focus of this study was to investigate whether nilotinib, a DDR1 inhibitor can inhibit breast cancer cells.

This study showed that nilotinib effectively blocked the proliferation of breast cancer cells and facilitated apoptosis. Further, the effect of nilotinib on the metabolism of breast cancer was assessed. Nilotinib showed a significant inhibitory effect on the migration of MCF-7 and MDA-MB-231 cells. Based on previous research reports, the impact of nilotinib on inhibition of DDR1 was investigated. As expected, nilotinib inhibited the phosphorylation of DDR1. Moreover, our results demonstrated that the downregulation of DDR1 by RNAi influenced the migration of MDA-MB-231 and
$\mathbf{A}$

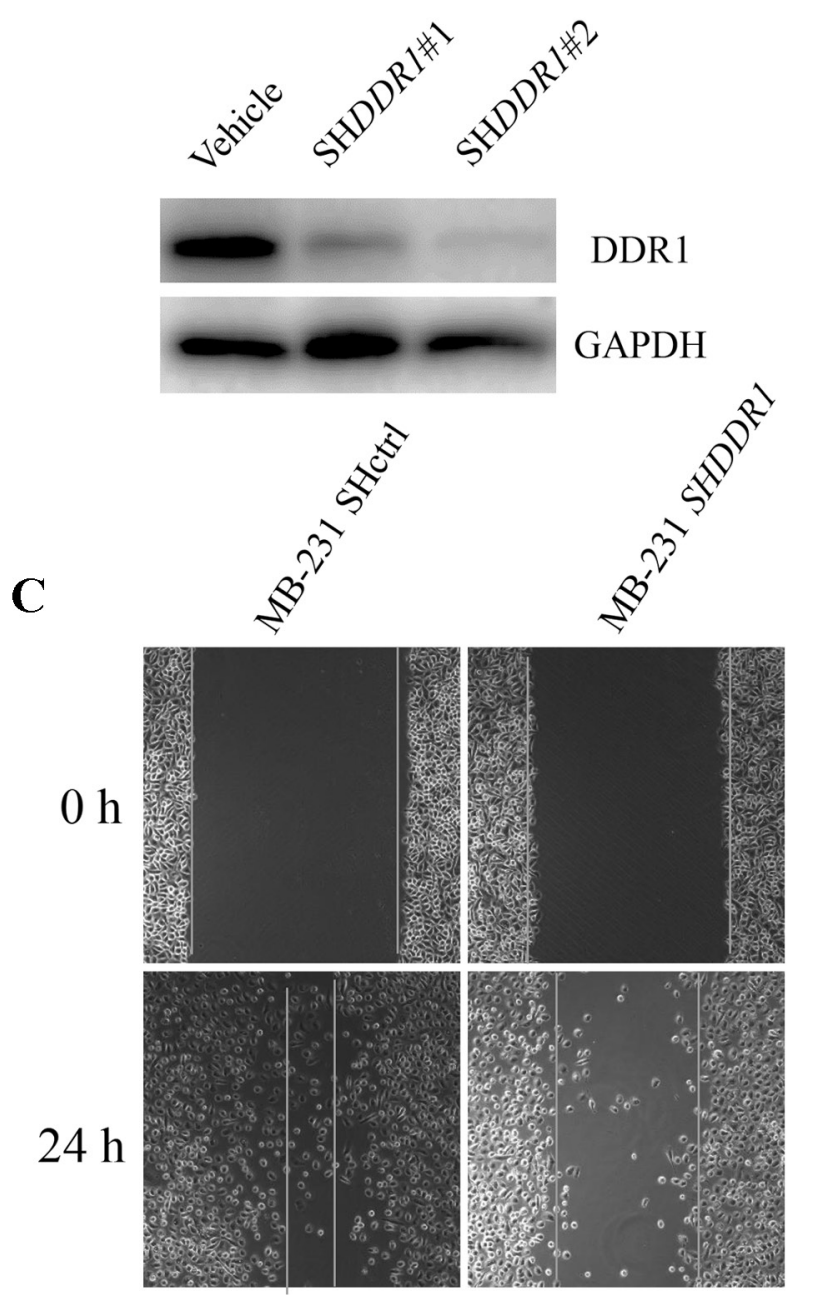

B

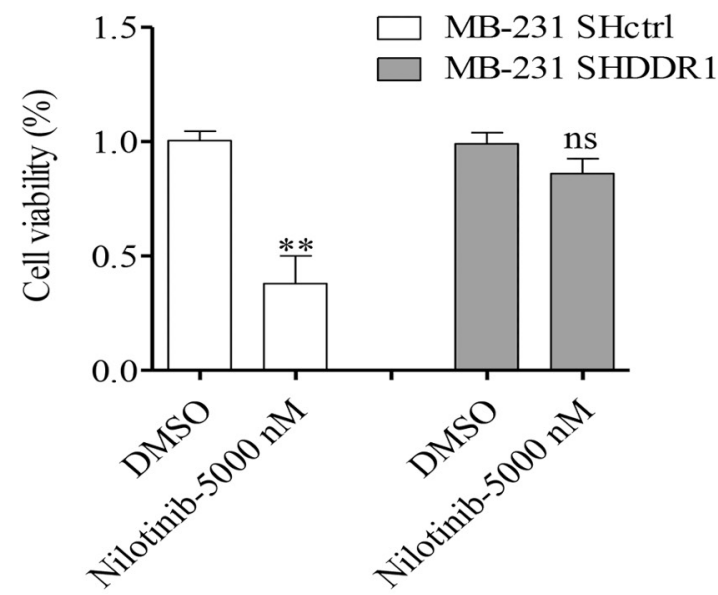

D

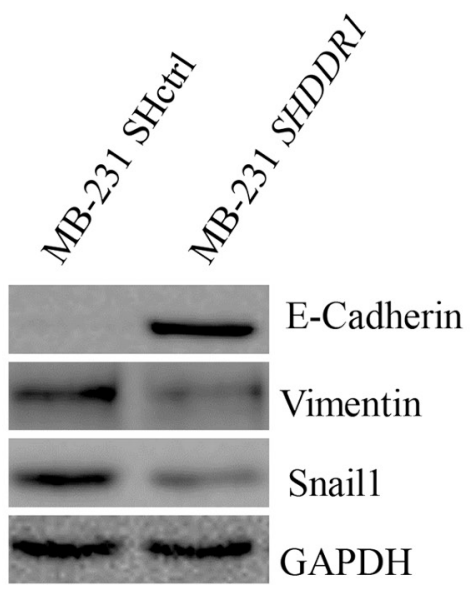

Figure 5. Effects of DDR1-shRNA on proliferation and migration of MDA-MB-231 cells. A) Effects of DDR1-shRNA on the protein expression. B) Comparison of the anti-proliferation ability of nilotinib (5000 nM) in MDA-MB-231 SHctrl and MDA-MB-231 SHDDR1 cells for 24 h. C) The different migration ability between MDA-MB-231 SHctrl and MDA-MB-231 SHDDR1 cells. D) Western blotting. Effects of DDR1-shRNA on protein expression levels of E-cadherin, Vimentin, and Snail1 in MDA-MB-231 cell lines. $\left({ }^{*} \mathrm{p}<0.05,{ }^{* *} \mathrm{p}<0.01\right.$, and ${ }^{* * *} \mathrm{p}<0.001$ compared to DMSO group). 
MCF-7 cells. This indicated the correlation between DDR1 and the metastatic ability of breast cancer cells. Subsequently, it was observed that nilotinib did not inhibit MDA-MB-231 SHDDR1 and MCF-7 SHDDR1 cells. The results indicated the inhibition of MDA-MB-231 and MCF-7 cells by nilotinib by targeting DDR1.

In a nutshell, DDR1 plays an essential role in the growth and migration of MCF-7 and MDA-MB-231 cells. Nilotinib inhibited MCF-7 and MDA-MB-231 cells by blocking the DDR1 signaling pathway. Therefore, targeting DDR1 therapeutically could potentially affect the survival and influence the metabolism of breast cancer, and nilotinib could be a potential candidate for the treatment of breast cancer.

Supplementary information is available in the online version of the paper.

Acknowledgments: Financial support was provided by the Natural Science Foundation of Ningbo (2018A610277), Zhejiang Provincial Department of Education General Research Project (Y201636648), including the design of the study and cost for experiments, analysis, interpretation of data.

\section{References}

[1] HANNA S, KHALIL B, NASRALLAH A, SAYKALI BA, SOBH R et al. StarD13 is a tumor suppressor in breast cancer that regulates cell motility and invasion. Int J Oncol 2014; 44: 1499-1511. https://doi.org/10.3892/ijo.2014.2330

[2] KAMATH R, MAHAJAN KS, ASHOK L, SANAL TS. A study on risk factors of breast cancer among patients attending the tertiary care hospital, in udupi district. Indian J Community Med 2013; 38: 95-99. https://doi.org/10.4103/09700218.112440

[3] KHALED N, BIDET Y. New Insights into the Implication of Epigenetic Alterations in the EMT of Triple Negative Breast Cancer. Cancers (Basel) 2019; 11: 559. https://doi. org/10.3390/cancers 11040559

[4] CAZZANigA ME, CAMERINI A, ADDEO R, NOLE F, MUNZONE E et al. Metronomic oral vinorelbine in advanced breast cancer and non-small-cell lung cancer: current statusand future development. Future Oncol 2016; 12: 373-387. https://doi.org/10.2217/fon.15.306

[5] TRUFFI M, SORRENTINO L, MAZZUCCHELLI S, FIANDRA L, CORSI F. What Is the Role of Nanotechnology in Diagnosis and Treatment of Metastatic Breast Cancer? Promising Scenarios for the Near Future. Journal of Nanomaterials 2016. https://doi.org/10.1155/2016/5436458

[6] MCGALE P, TAYLOR C, CORREA C, CUTTER D, DUANE $\mathrm{F}$ et al. Effect of radiotherapy after mastectomy and axillary surgery on 10-year recurrence and 20-year breast cancer mortality: meta-analysis of individual patient data for 8135 women in 22 randomised trials. Lancet 2014; 383: $2127-$ 2135. https://doi.org/10.1016/S0140-6736(14)60488-8
[7] SISTI JS, COLLINS LC, BECK AH, TAMIMI RM, ROSNER BA et al. Reproductive risk factors in relation to molecular subtypes of breast cancer: Results from the nurses' health studies. Int J Cancer 2016; 138: 2346-2356. https:// doi.org/10.1002/ijc.29968

[8] YEH YC, LIN HH, TANG MJ. Dichotomy of the function of DDR1 in cells and disease progression. Biochim Biophys Acta Mol Cell Res 2019; 1866: 118473. https://doi. org/10.1016/j.bbamcr.2019.04.003

[9] ONGUSAHA PP, KIM JI, FANG L, WONG TW, YANCOPOULOS GD et al. p53 induction and activation of DDR1 kinase counteract p53-mediated apoptosis and influence p53 regulation through a positive feedback loop. EMBO J 2003; 22: 1289-1301. https://doi.org/10.1093/emboj/cdg129

[10] RUIZ PA, JARAI G. Discoidin domain receptors regulate the migration of primary human lung fibroblasts through collagen matrices. Fibrogenesis Tissue Repair 2012; 5: 3. https:// doi.org/10.1186/1755-1536-5-3

[11] BELFIORE A, MALAGUARNERA R, NICOLOSI ML, LAPPANO R, RAGUSA $M$ et al. A novel functional crosstalk between DDR1 and the IGF axis and its relevance for breast cancer. Cell Adh Migr 2018; 12: 305-314. https://doi. org/10.1080/19336918.2018.1445953

[12] HU Y, LIU J, JIANG B, CHEN J, FU Z et al. MiR-199a-5p loss up-regulated DDR1 aggravated colorectal cancer by activating epithelial-to-mesenchymal transition related signaling. Dig Dis Sci 2014; 59: 2163-2172. https://doi.org/10.1007/ s10620-014-3136-0

[13] HUO Y, YANG M, LIU W, YANG J, FU X et al. High expression of DDR1 is associated with the poor prognosis in Chinese patients with pancreatic ductal adenocarcinoma. J Exp Clin Cancer Res 2015; 34: 88. https://doi.org/10.1186/ s13046-015-0202-1

[14] CASTRO-SANCHEZ L, SOTO-GUZMAN A, GUADERRAMA-DIAZ M, CORTES-REYNOSA P, SALAZAR EP et al. Role of DDR1 in the gelatinases secretion induced by native type IV collagen in MDA-MB-231 breast cancer cells. Clin Exp Metastasis 2011; 28: 463-477. https://doi. org/10.1007/s10585-011-9385-9

[15] CASTRO-SANCHEZ L, SOTO-GUZMAN A, NAVARRO-TITO N, MARTINEZ-OROZCO R, SALAZAR EP et al. Native type IV collagen induces cell migration through a CD9 and DDR1-dependent pathway in MDA-MB-231 breast cancer cells. Eur J Cell Biol 2010; 89: 843-852. https:// doi.org/10.1016/j.ejcb.2010.07.004

[16] AZIZI R, SALEMI Z, FALLAHIAN F, AGHAEI M. Inhibition of didscoidin domain receptor 1 reduces epithelial-mesenchymal transition and induce cell-cycle arrest and apoptosis in prostate cancer cell lines. J Cell Physiol 2019; 234: 19539-19552. https://doi.org/10.1002/jcp.28552

[17] CHEN HR, YEH YC, LIU CY, WU YT, LO FY et al. DDR1 promotes E-cadherin stability via inhibition of integrin-beta1-Src activation-mediated E-cadherin endocytosis. Sci Rep 2016; 6: 36336. https://doi.org/10.1038/srep36336

[18] DAY E, WATERS B, SPIEGEL K, ALNADAF T, MANLEY $\mathrm{PW}$ et al. Inhibition of collagen-induced discoidin domain receptor 1 and 2 activation by imatinib, nilotinib and dasatinib. Eur J Pharmacol 2008; 599: 44-53. https://doi. org/10.1016/j.ejphar.2008.10.014 
[19] SAYDAM G, HAZNEDAROGLU IC, KAYNAR L, YAVUZ AS, ALI $\mathrm{R}$ et al. Frontline nilotinib treatment in Turkish patients with Philadelphia chromosome-positive chronic Myeloid Leukemia in chronic phase: updated results with 2 years of follow-up. Hematology 2018; 23: 771-777. https:// doi.org/10.1080/10245332.2018.1498167

[20] JEITANY M, LEROY C, TOSTI P, LAFITTE M, LE GUET $\mathrm{J}$ et al. Inhibition of DDR1-BCR signalling by nilotinib as a new therapeutic strategy for metastatic colorectal cancer. EMBO Mol Med 2018; 10: e7918. https://doi.org/10.15252/ emmm.201707918

[21] HOCHHAUS A, SAGLIO G, HUGHES TP, LARSON RA, KIM DW et al. Long-term benefits and risks of frontline nilotinib vs imatinib for chronic myeloid leukemia in chronic phase: 5-year update of the randomized ENESTnd trial. Leukemia 2016; 30: 1044-1054. https://doi.org/10.1038/ leu. 2016.5
[22] RIX U, HANTSCHEL O, DUERNBERGER G, RIX LLR, PLANYAVSKY $M$ et al. Chemical proteomic profiles of the BCR-ABL inhibitors imatinib, nilotinib, and dasatinib, reveal novel kinase and nonkinase targets. Blood 2007; 110: 4055-4063. https://doi.org/10.1182/blood-2007-07-102061

[23] MEIRSON T, GENNA A, LUKIC N, MAKHNII T, ALTER $\mathrm{J}$ et al. Targeting invadopodia-mediated breast cancer metastasis by using ABL kinase inhibitors. Oncotarget 2018; 9: 22158-22183. https://doi.org/10.18632/oncotarget.25243

[24] SUN H, CAO D, WU H, LIU H, KE X et al. Development of low molecular weight heparin based nanoparticles for metastatic breast cancer therapy. Int J Biol Macromol 2018; 112: 343-355. https://doi.org/10.1016/j.ijbiomac.2018.01.195

[25] SCULLY OJ, BAY BH, YIP G, YU Y. Breast cancer metastasis. Cancer Genomics Proteomics 2012; 9: 311-320.

[26] GIULIANO AE, HUNT KK, BALLMAN KV, BEITSCH PD, WHITWORTH PW et al. Axillary dissection vs no axillary dissection in women with invasive breast cancer and sentinel node metastasis: a randomized clinical trial. JAMA 2011; 305: 569-575. https://doi.org/10.1001/jama.2011.90 


\section{Nilotinib, a discoidin domain receptor 1 (DDR1) inhibitor, induces apoptosis and inhibits migration in breast cancer}

Shuai $\mathrm{WANG}^{1}$, Yanping $\mathrm{XIE}^{1}$, Aina BAO ${ }^{1}$, Jing $\mathrm{LI}^{1}$, Tingting $\mathrm{YE}^{1}$, Chu YANG ${ }^{1}$, Shufang $\mathrm{YU}^{2, *}$

Supplementary Information

A

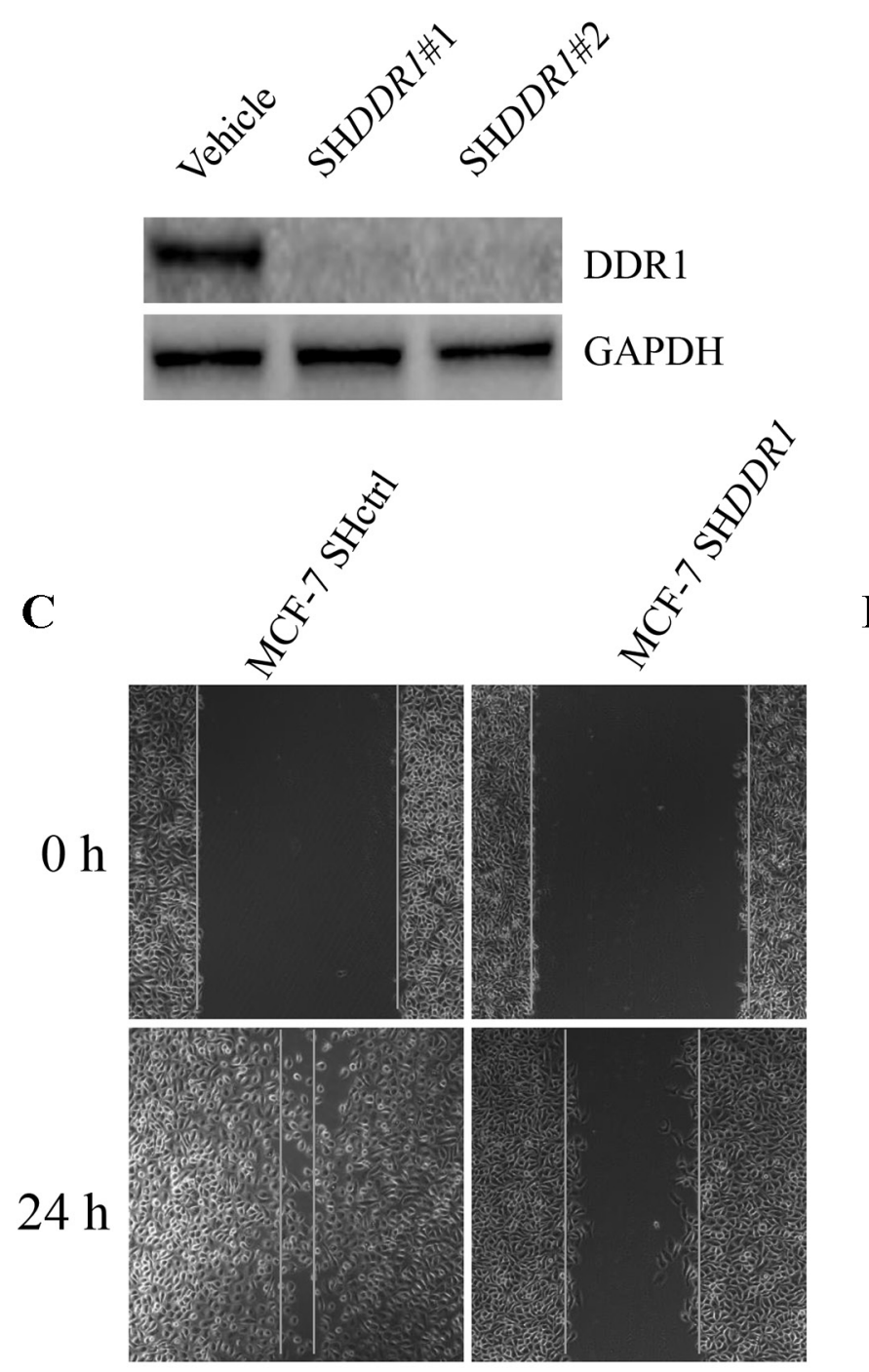

B

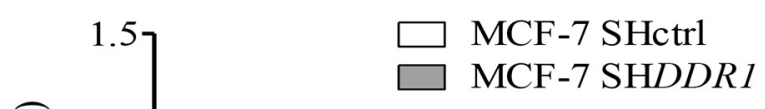

$\mathbf{D}$
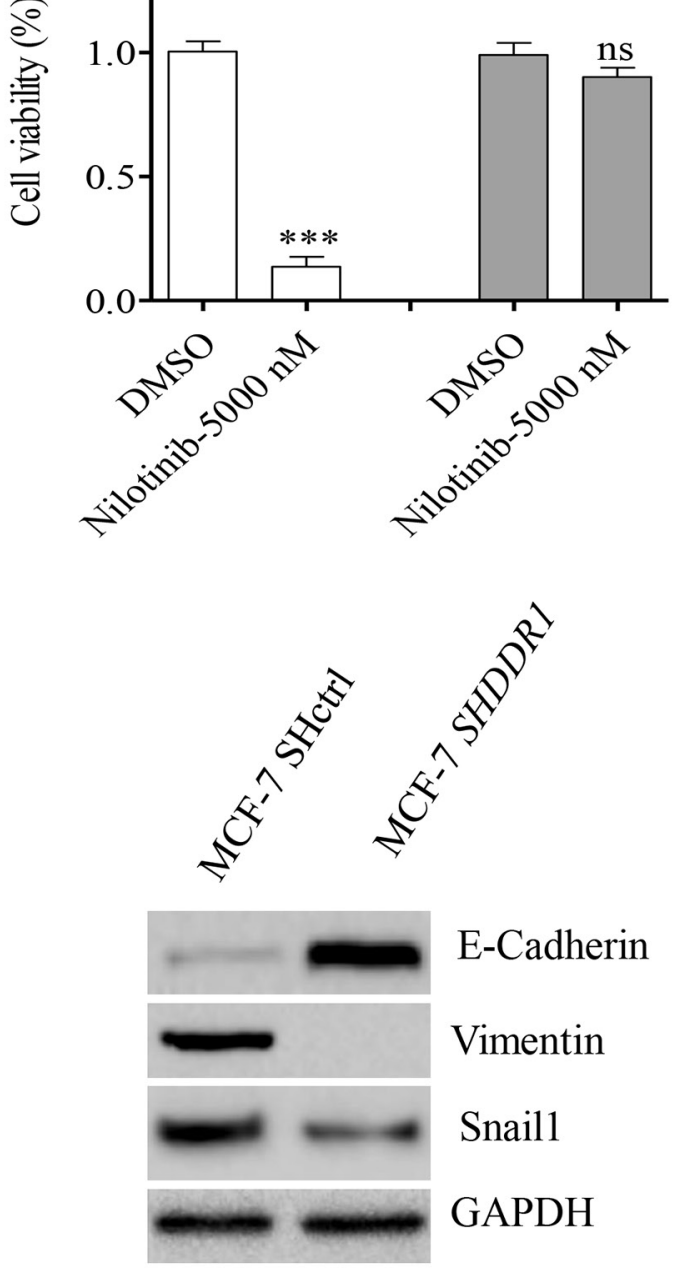

Supplementary Figure S1. Effects of DDR1-shRNA on proliferation and migration MCF-7. A) Effects of DDR1-shRNA on expression of protein. B) Comparison of the anti-proliferation ability of Nilotinib (5,000 nM) to MCF-7 SHctrl and MCF-7 SHDDR1 for $24 \mathrm{~h}$. C) The different migration ability between MCF-7 SHctrl and MCF-7 SHDDR1. D) Western blotting. Effects of DDR1-shRNA on protein expression levels of E-Cadherin, Vimentin, and Snail1 in MCF-7 cell lines. $\left({ }^{*} \mathrm{p}<0.05,{ }^{* *} \mathrm{p}<0.01\right.$ and ${ }^{* * *} \mathrm{p}<0.001$ compared to DMSO group). 


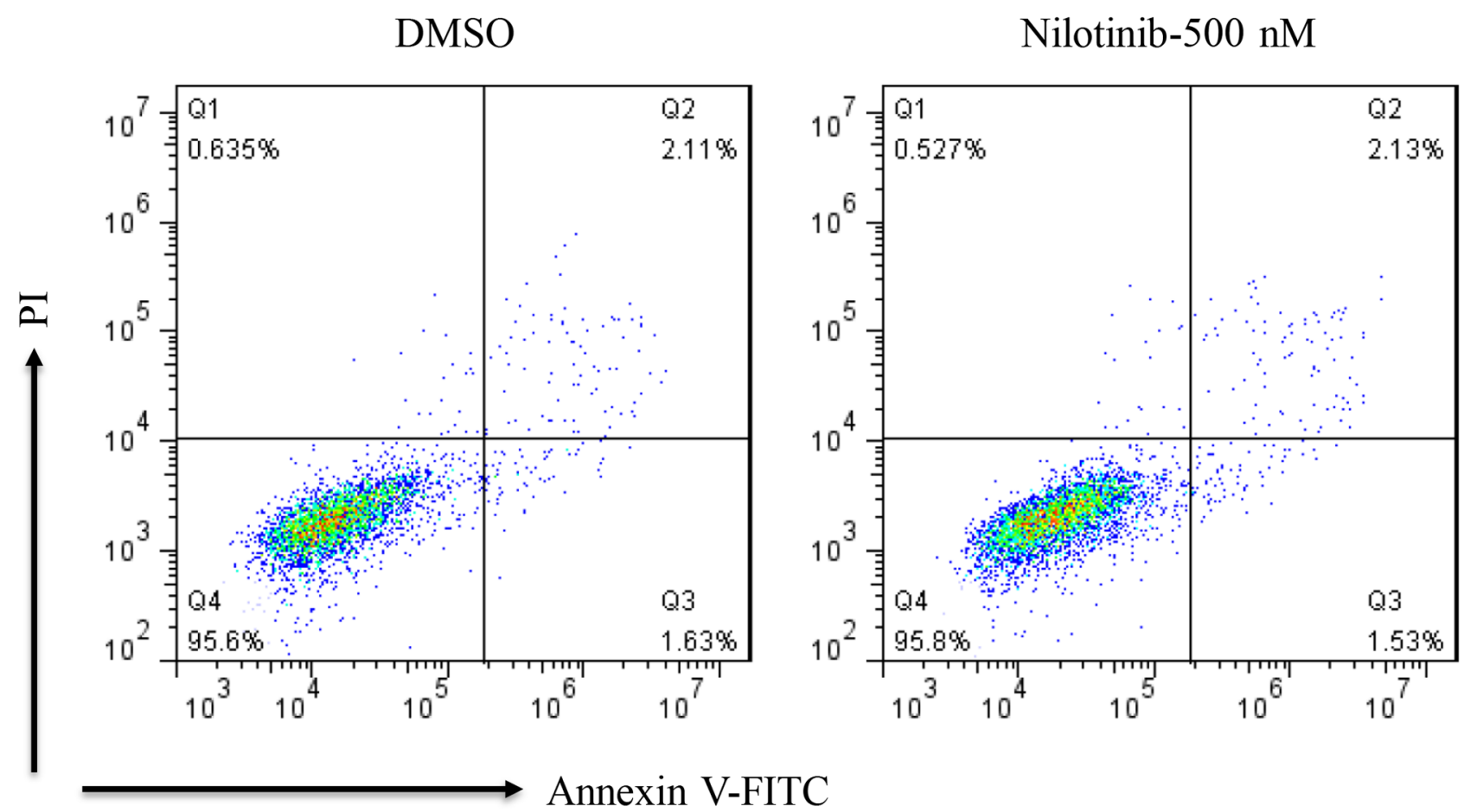

Supplementary Figure S2. Flow cytometry, effort of Nilotinib (500 nM) on apoptosis in MDA-MB-231 SHDDR1. 